\title{
AN APPROXIMATION METHOD FOR SIMULATING TEMPERATURE DEPENDENCE OF POISSON'S RATIOS OF SELF-EXPANDING AUXEGENS
}

\author{
HONGMEI WU* AND GAOYUAN WEI** \\ Department of Polymer Science and Engineering, College of Chemistry and Molecular Engineering \\ Peking University, Beijing 100871, People's Republic of China
}

(Rec. 13 December 2004)

PACS 62.20.D, 71.20.R, 05.65, 68.65

\begin{abstract}
A combined molecular dynamics and molecular mechanics method has been developed for estimating Poisson's ratios of certain types of molecular auxetics at various temperatures. The temperature dependence of the auxeticity of a special class of molecular auxetics, namely, a self-expanding supramolecular network of auxegens containing alternating phenyl and acetylene links, is studied with use of this approximation method. The simulation results show that as temperature increases from 0 to $300 \mathrm{~K}$, the auxeticity of the resulting superlattice or van der Waals network of auxegens decreases from the initial self-expandability to two negatively small Poisson ratios on the xoy plane.
\end{abstract}

Key words: Poisson ratio, polymers, self-organized systems, superlattices, auxetics, auxegen, auxeticity

\section{INTRODUCTION}

Computer simulation of auxetic behaviours of molecular auxetics has long been an important area of auxetic research [1,2] since the pioneering work of Wojciechowski [3-5] and Evans et al. [6-8]. In 2000, Grima and Evans [9] reported a 2D triangular polyphenylacetylene network which was shown by molecular mechanics calculation to be self-expandable, i.e., having six negative Poisson's ratios, as were the case for a superlattice of auxegens connected through van der Waals interactions, which was discovered much earlier in our laboratory, but never published. And Wei [10] recently presented a general method of designing auxetic selfassemblies of polymers with alternating 4-functionality double-arrow hard blocks and flexible spacers, while other kinds of auxetic supramolecules were also modelled by us $[11,12]$. In this paper, we first present an approximation method, which combines both molecular dynamics (MD) and molecular mechanics (MM) results, to study the temperature dependence of Poisson's ratios of certain types of molecular auxetics. We then apply the method developed to a self-expandable van der Waals network of special polyphenylacetylene auxegens with

\footnotetext{
* Present address: Beijing Research Institute of Chemical Industry, SINOPEC, Beijing 100013, P. R. China.

** Corresponding author: e-mail: gywei@pku.edu.cn, phone: 86-10-62754177, fax: 86-10-62751708.
} 
the dependence of its auxeticity on temperature given. We finally give a discussion of both the method and the results and draw some conclusions from the discussion.

\section{METHODOLOGY AND SIMULATION RESULTS}

It is well known that in a MD simulation the internal stress tensor for the molecular system can be obtained using the so-called virial expression which relates the stress to not only the atomic positions but also the atomic velocities [13]. Often some fluctuation formulae applied to simulations in different ensembles are then used for obtaining the elastic stiffness coefficients, which implicitly captures entropic effects. The simplest formula, applicable when simulations have been performed in the constant stress ensemble, is that of Parrinello and Rahman [14]. Another fluctuation formula is due to Gusev et al. [15], which can significantly improve the convergence behavior over the Parrinello and Rahman formula. Finally, from the obtained stiffness coefficients, the anisotropic Poisson's ratios may be approximately estimated. However, in cases where only approximate values of the anisotropic Poisson's ratios are needed such as in our studies of theoretical or yet-to-be-synthesized molecular auxetics, one may employ a much more efficient approximation method which will be described below.

The crucial condition for our approximation method to hold is that the lowest-total-energy molecular structure from an MD simulation does not undergo any appreciable change in the structure itself when a further potential energy minimization is done on the structure. Under this condition, the mechanical properties calculated from the final minimized structure at $0 \mathrm{~K}$ may be regarded as an approximation to those of the structure at the same temperature as used in the MD simulation. In what follows, this method will be employed to study the temperature dependence of Poisson's ratios of certain types of molecular auxetics, i.e., a selfexpandable van der Waals network of special polyphenylacetylene auxegens, whose repeating unit cell is shown in Fig. 1. The forcefield used in obtaining the minimized structure in Fig. 1 is Dreiding 2.21 suitable for general organic compounds [16]. The self-expandability of this structure is then revealed through its six negative Poisson ratios (see Table 1) calculated with use of the Second Derivative Method of the Mechanical Properties Module of the Accelrys Cerius $^{2} 4.2$ software package.

To see if this self-expandability will survive at a higher temperature, we ran a series of MD simulations for different temperatures: 200, 240, 280, 300, and $340 \mathrm{~K}$, and the resulting lowest-total-energy molecular structures are displayed in Figs. 2 and 3. The time step used is $1 \mathrm{fs}$ as the fast movement of this kind of auxegens is the vibration of $\mathrm{C}-\mathrm{H}$ stretch. There are a total of 360 atoms in the molecular auxegen contained in the unit cell of the superlattice. The velocity scaling method is applied to calculate the structures at any given temperature within the constant-temperature, constant-pressure ensemble (NPT) for 200 ps. The lowest-totalenergy molecular structures in Fig. 2 are then minimized using MM, and the so-minimized structures are further compared with the starting ones from the MD simulations (see Figs. 4-7). 

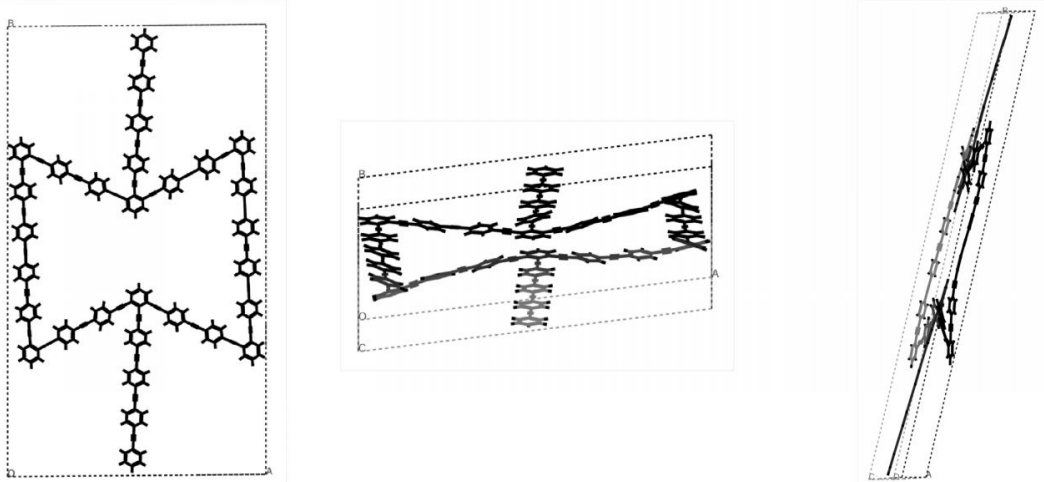

Fig. 1. A minimized structure of the self-expandable van der Waals network of polyphenylacetylene auxegens. From left to right: front view (xoy plane), top view ( $x o z \backslash$ and right-side view (yoz)
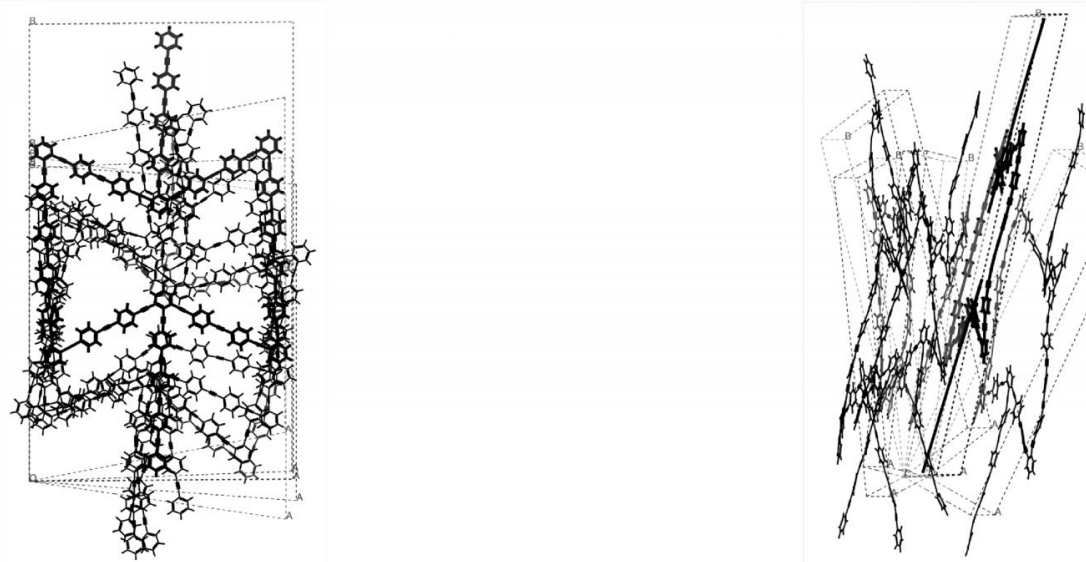

Fig. 2. The lowest-total-energy structures of the self-expandable van der Waals network of polyphenylacetylene auxegens from MD simulations for different temperatures: 0, 200, 240, 280, and $300 \mathrm{~K}$. From left to right: front view (xoy plane), and right-side view (yoz)

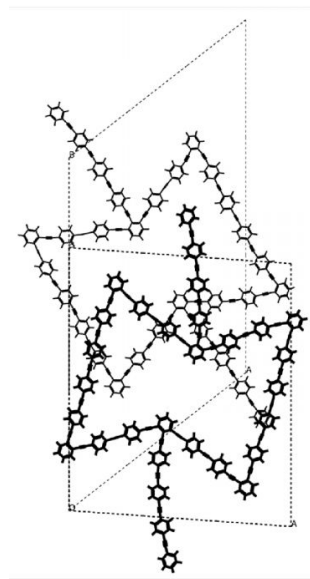

Fig. 3. The front view (xoy plane) of the lowest-total-energy structures of the self-expandable van der Waals network of polyphenylacetylene auxegens from MD simulations for two different temperatures: 300 and $340 \mathrm{~K}$ 

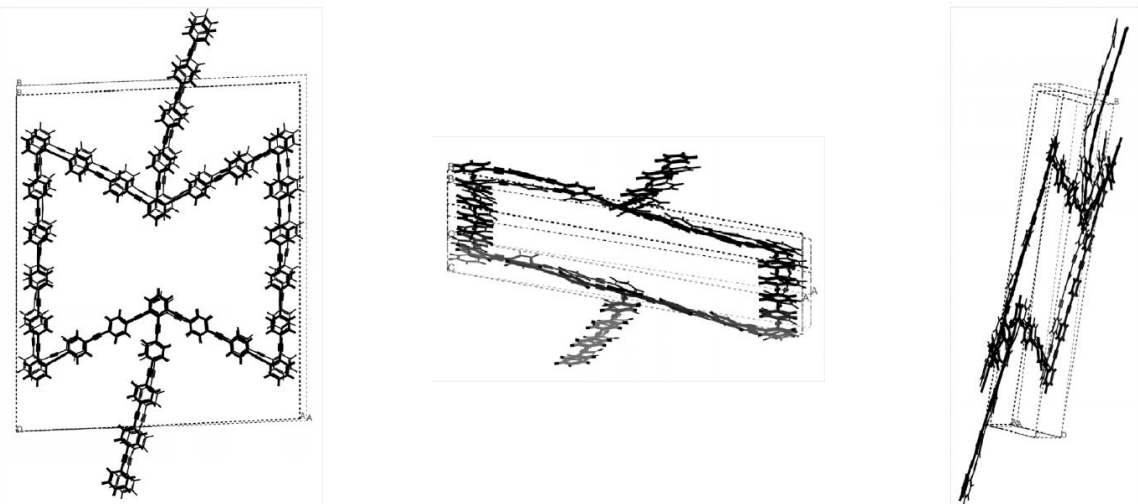

Fig. 4. A comparison of the MM minimized structure with the starting one from the MD simulation at $200 \mathrm{~K}$ for the superlattice of poly-phenyl-acetylene auxegens. From left to right: front view (xoy plane), top view (xoz), and right-side view (yoz)
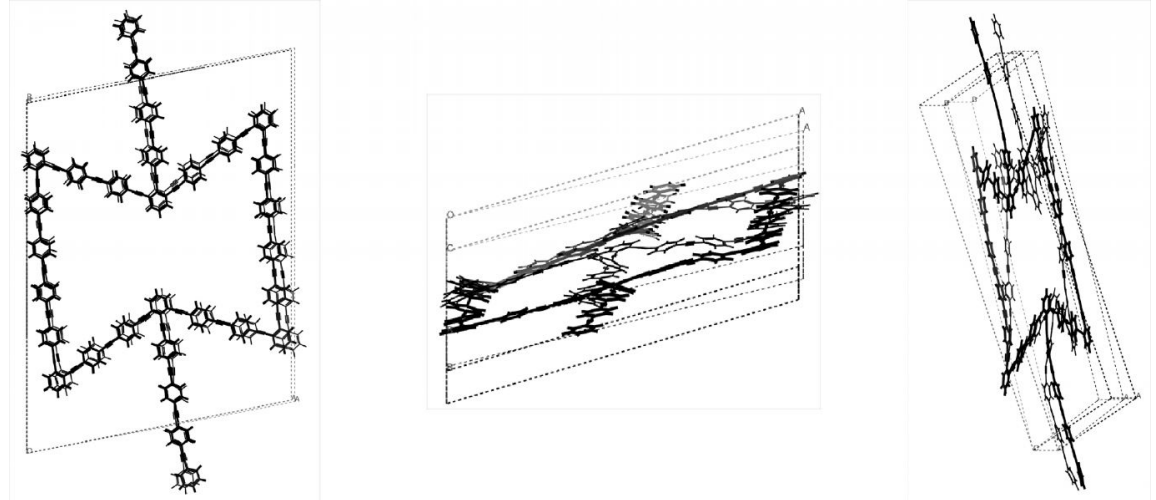

Fig. 5. A comparison of the MM minimized structure with the starting one from the MD simulation at 240K for the superlattice of poly-phenyl-acetylene auxegens. From left to right: front view (xoy plane), top view (xoz), and right-side view (yoz)
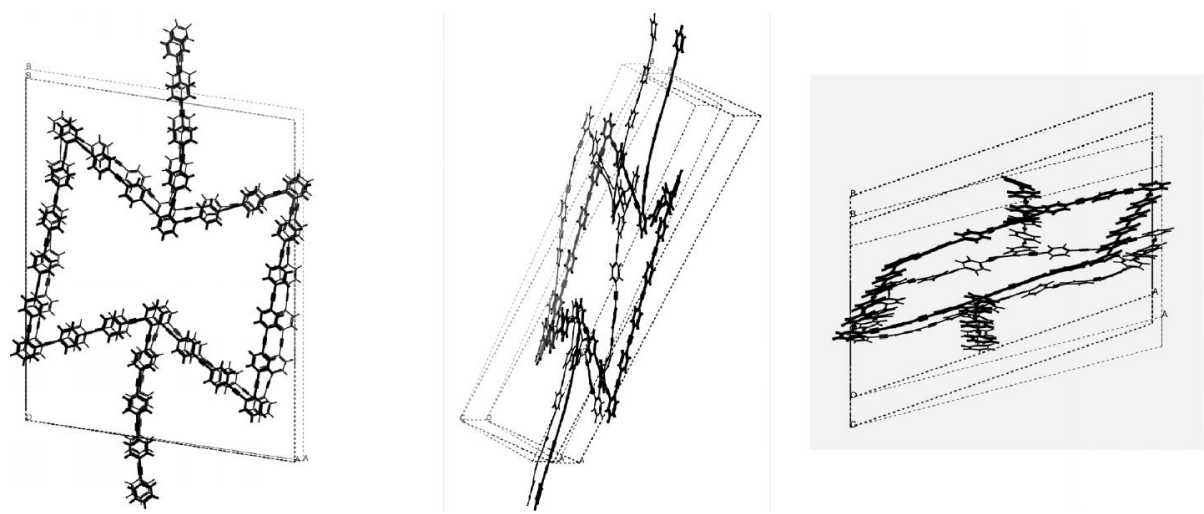

Fig. 6. A comparison of the MM minimized structure with the starting one from the MD simulation at $280 \mathrm{~K}$ for the superlattice of poly-phenyl-acetylene auxegens. From left to right: front view (xoy plane), right-side view (yoz), and top view (xoz) 

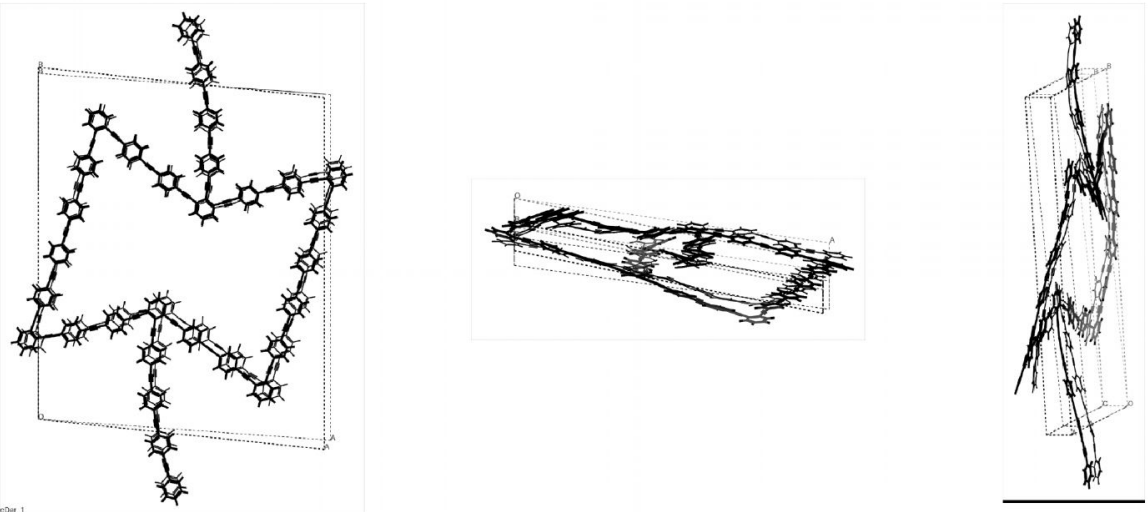

Fig. 7. A comparison of the MM minimized structure with the starting one from the MD simulation at $300 \mathrm{~K}$ for the super-lattice of poly-phenyl-acetylene auxegens. From left to right: front view (xoy plane), top view ( $x o z)$, and right-side view (yoz)

Table 1. The temperature-dependence of Poisson ratios of a van der Waals network of poly-phenylacetylene auxegens as approximately estimated from MD simulations for different temperatures: 0,200 , $240,280,300$, and $340 \mathrm{~K}$ counted from the top to the bottom data row

\begin{tabular}{rrrrrr}
\hline$\sigma_{x y}$ & $\sigma_{y x}$ & $\sigma_{x z}$ & $\sigma_{z x}$ & $\sigma_{y z}$ & $\sigma_{z y}$ \\
\hline-1.1199 & -0.5599 & -0.0446 & -0.1730 & -0.0239 & -0.1855 \\
-0.5832 & -0.3052 & 0.1266 & 0.2488 & 0.0847 & 0.3181 \\
-0.4921 & -0.2059 & 0.1000 & 0.2170 & 0.0185 & 0.0961 \\
-0.3261 & -0.1709 & 0.2023 & 0.5808 & 0.0514 & 0.2815 \\
-0.1440 & -0.0686 & 0.0999 & 0.1999 & 0.0408 & 0.1713 \\
0.0828 & 0.1246 & 0.0480 & 0.1808 & 0.0842 & 0.2108 \\
\hline
\end{tabular}

Fortunately the MM minimized molecular structures are not appreciably different from the corresponding lowest-total-energy ones of the MD simulations, and we may then use the minimized structures for different non-zero temperatures to evaluate approximately the anisotropic Poisson ratios as done for the $0 \mathrm{~K}$ case described above. The results are tabulated in Table 1 .

\section{DISCUSSION AND CONCLUSION}

The MM and MD results in previous section indicates that there exists indeed some molecular system for which the condition for our approximation method is met (see especially Figs. 4-7). From Figs. 2 and 3, it is seen that the rise in temperature changes the optimum packing geometry of both auxegen and the associated superlattice or van der Waals network, thus resulting in the decrease in the auxeticity as shown in Table 1. The self-expandability of the van der Waals network of polyphenylacetylene auxegens at $0 \mathrm{~K}$ is also lost as temperature rises to above $0 \mathrm{~K}$. Furthermore, although our results on the temperature-dependence of the auxeticity of the auxegens is obtained by use of an approximation method and hence is 
approximate only, the trend that our results reveals is nevertheless reasonable because as the temperature increases the molecular movement will become faster and the concordant effect of all the atoms to acquire a negative Poisson ratio will become more difficult. Especially the upper temperature limit for our van der Waals network to be auxetic is $300 \mathrm{~K}$, which is a common choice of temperature for most synthetic work and for most daily uses of the auxetic network materials. The reason that the disappearance of auxeticity occurs at some temperature between 300 and $340 \mathrm{~K}$ may be seen from Fig. 3 which shows that there is a sharp change in the packing geometry. It is interesting to note here that the auxegens studied in this paper is a second published example of self-expandable molecular auxetics at $0 \mathrm{~K}$ [9], and that a nonzero-temperature self-expandable molecular auxetic is yet to be found.

In conclusion, we have developed an approximation method for simulating temperaturedependence of auxeticity or elasticity of any molecular auxetics or non-auxetics. When applied to a van der Waals network of polyphenylacetylene auxegens, our approximation method gives results that indicates that the auxeticity deceases with the increase in temperature and that the self-expandability at $0 \mathrm{~K}$ disappears at temperature above $0 \mathrm{~K}$. This finding may explain the fact that the room-temperature auxeticity of any molecular auxetics is indeed very difficult to achieve, and it may thus be pointed out that the molecular design or modelling work must be aimed at those auxegens or auxetics with the $0 \mathrm{~K}$ Poisson ratios as completely negative and negatively high as possible in order for a man-made molecular auxetic to appear.

\section{Acknowledgments}

We gratefully acknowledge the financial support from the NSFC through the grants 20274004 and 29574150, the P\&G Asia, and The Royal Society K C Wong Foundation.

\section{References}

[1] K. E. Evans and A. Alderson, Adv. Mater. 12, 617 (2000).

[2] G. Y. Wei, Mater. Rev. (in Chinese) 17, 8 (2003).

[3] K. W. Wojciechowski, Mol. Phys. 61, 1247 (1987).

[4] K. W. Wojciechowski, Phys. Lett. A 137, 60 (1989).

[5] K. W. Wojciechowski, K. V. Tretiakov, and M. Kowalik, Phys. Rev. E 67, 036121 (2003).

[6] K. E. Evans, M. A. Nkansah, I. J. Hutchinson, and S. C. Rogers, Nature 353, 124 (1991).

[7] G. Y. Wei, S. F. Edwards, I. J. Hutchinson, W. C. Mackrodt, S. C. Rogers, and K E. Evans, Research Report No. I C 15657 (ICI Chemicals and Polymers, Runcorn, UK) on A Feasibility Study on the Chemical Synthesis of 'Auxetic' Materials - A Novel Class of Molecular Networks with -ve Poisson Ratios, 2 September 1991. G. Y. Wei, S. F. Edwards, Research Proposal to ICI Chemicals and Polymers (Runcorn, UK) - Synthesis ofNegative-Poisson-Ratio Molecular Networks, 8 November 1990.

[8] K. E. Evans, A. Alderson, and F. R. Christian, J. Chem. Soc. Faraday Trans. 91, 2671 (1995).

[9] J. N. Grima and K. E. Evans, Chem. Commun. 1531 (2000).

[10] G. Y. Wei, Phys. Stat. Sol. B (in press).

[11] H. M. Wu and G. Y. Wei, Acta Polvm. Sinica (2), 198 (2004).

[12] H. M. Wu and G. Y. Wei, Chin. J. Polym. Sci. 22(4), 355 (2004).

[13] J. H. Weiner, Statistical Mechanics of Elasticity, John Wiley: New York (1983).

[14] M. Parrinello and A. Rahman, J. Chem. Phys. 76, 2662 (1982).

[15] A. A. Gusev, M. M. Zehnder, U. W. Suter, Phys. Rev. Rep. B 54, 1 (1996).

[16] Cerius ${ }^{2} 4.0$ User Guide, Property Prediction, Molecular Simulations Inc., San Diego (1999). 